\title{
Increased production of luminol enhanced chemiluminescence by the inflamed colonic mucosa in patients with ulcerative colitis
}

S Sedghi, J Z Fields, M Klamut, G Urban, M Durkin, D Winship, D Fretland, M Olyaee, A Keshavarzian
Departments of Medicine and Pharmacology, Loyola University Medical Center, Maywood, IL; Medical and Research Services, Hines VA Hospital, Hines, IL

S Sedghi

J Z Fields

M Klamut

G Urban

M Durkin

D Winship

M Olyaee

A Keshavarzian

Searle Laboratories, Skokie, IL

D Fretland

Correspondence to: Dr A Keshavarzian, Professor of Medicine and Pharmacology, Division of Digestive Diseases and Nutrition, Loyola University Medical Center, 2160 South First Avenue, Maywood, IL 60153, USA

Accepted for publication 5 January 1993

\begin{abstract}
Reactive oxygen species have been implicated as mediators of inflammation in ulcerative colitis. Chemiluminescence is a reliable means of estimating reactive oxygen species in biological media. Increased reactive oxygen species values in the inflamed colonic mucosa in rats were seen by chemiluminescence. The aims of the study were to find out if chemiluminescence is raised in the colonic mucosa of patients with ulcerative colitis and correlates with disease activity, and to elucidate the sources of the chemiluminescence. It was found that reactive oxygen species, as measured by the chemiluminescence technique, are raised in inflamed colonic mucosa and correlates with symptom score, sigmoidoscopic score, disease activity, and activity of the neutrophil enzyme myeloperoxidase. Chemiluminescence was inhibited by a myeloperoxidase inhibitor (azide) and an $\mathrm{H}_{2} \mathrm{O}_{2}$ scavenger (catalase) but not by allopurinol, an inhibitor of the enzyme xanthine oxidase. Chemiluminescence was also inhibited by indomethacin, but this did not seem to be related to inhibition of cyclo-oxygenase. These findings suggest that a likely cellular source of reactive oxygen species in the inflamed colon of patients with ulcerative colitis is the neutrophil and that myeloperoxidase conversion of $\mathrm{H}_{2} \mathrm{O}_{2}$ to hypochlorous acid, contributes to the chemiluminescence signal and possibly, to the tissue injury. Neither cyclo-oxygenase nor lipoxygenase seem to play a part as sources for the chemiluminescence.

(Gut 1993; 34: 1191-1197)
\end{abstract}

Although the cause of ulcerative colitis is unclear, some information is available on its pathophysiology, including several proposed mediators of the inflammation and tissue injury in this disease. ${ }^{12}$ Among such factors are the group of chemicals known as reactive oxygen species that are final common mediators of inflammation and tissue injury in general, ${ }^{34}$ and are also thought to play a part in ulcerative colitis. $^{5-8}$

Chemiluminescence is a simple, sensitive, and reproducible technique that can be used to estimate reactive oxygen species in biological media..$^{9-12}$ Using chemiluminescence produced by cells and extracts, ${ }^{10-12}$ several investigators have showed the role of reactive oxygen species in different disorders. In contrast, reports on the use of this technique for tissue reactive oxygen species are very limited. ${ }^{913-15}$ Using luminol enhanced chemiluminescence of colonic mucosa, we ${ }^{16}$ and Simmonds $e t a l^{17}$ have recently shown that reactive oxygen species values are raised in the inflamed colonic mucosa in patients with ulcerative colitis. These reports followed our earlier study in which we reported similar findings in animals with experimentally induced colonic inflammation. ${ }^{16} 18$

One potential source for the increased values of reactive oxygen species in inflammatory bowel disease is the neutrophil. These cells infiltrate into the colonic mucosa and are abundant during the active phase of colitis. ${ }^{1219}$ Neutrophils are capable of producing superoxide and a cascade of various reactive oxygen species leading to $\mathrm{H}_{2} \mathrm{O}_{2}$ and then, through the myeloperoxidase reaction, to hypochlorous acid. ${ }^{36}$

A second possible source of reactive oxygen species is the xanthine oxidase pathway in colonocytes that also produces superoxide anions by conversion of xanthine/hypoxanthine to uric acid. ${ }^{5021} \mathrm{~A}$ third possible source of superoxide anions and $\mathrm{H}_{2} \mathrm{O}_{2}$ is the oxidation of arachidonic acid through either the lipoxygenase reaction (producing leukotrienes) or the cyclo-oxygenase reaction (producing prostaglandins). ${ }^{152-24}$ This superoxide could then be metabolised further to other reactive oxygen species either in colonocytes or in neutrophils.

Accordingly, the aims of this study were (1) to confirm our previous preliminary finding ${ }^{16}$ that chemiluminescence is increased in colonic tissue from humans with active colitis, (2) to determine the cellular and biochemical sources of this chemiluminescence, (3) to identify the particular reactive oxygen species species involved, and (4) to find out if mucosal chemiluminescence is a reliable index of disease activity.

\section{Methods}

DESCRIPTION OF SUBJECT POPULATION

The study population consisted of 11 men and 16 women who were not pregnant with a mean age of 41 (range 21 to 70). All had a diagnosis of ulcerative colitis confirmed by air contrast barium enema, sigmoidoscopy or colonoscopy, with previous rectal or colonoscopic biopsies. Patients with active colitis had clinical symptoms of urgency, loose stools, abdominal pain, and blood in the stool. These patients were receiving no treatment at the time of mucosal biopsy Patients with inactive colitis had no urgency or rectal bleeding and had normal sigmoidoscopic findings. Their usual drugs, which were either 
sulphasalazine or oral 5-aminosalicylic acid (5-ASA), were stopped at least 48 hours before mucosal biopsy specimens were taken.

Patients had no cardiac, renal, hepatic, endocrine or metabolic disease - only ulcerative colitis and its sequelae. Before enrollment into the study, all patients had stopped taking prednisone for at least three weeks, immunosuppressive drugs for at least three months, and sulfasalazine and oral 5-ASA for at least 48 hours.

The control population consisted of 14 volunteer subjects (nine men, five women) with mean age of 44 (range 22 to 72 ) each of whom had a colonoscopic examination with normal results. These subjects had an examination for evaluation of occult blood, positive stools for screening for colon cancer, or both. To clarify whether the effects of indomethacin were through cyclooxygenase, we obtained colonic pinch biopsy specimens from an additional group of six patients with ulcerative colitis for an indomethacin dose response assay.

These studies were approved by the Institutional Review Board for the Protection of Human Subjects at Loyola University Medical Center. All subjects had voluntarily given written informed consent before the start of the study.

\section{ASSESSMENT OF DISEASE ACTIVITY BY SYMPTOM RATING AND SIGMOIDOSCOPY}

Symptoms - Symptoms were graded according to the presence and severity of diarrhoea $(0=$ formed stool; $1=$ occasional liquid stool; $2=$ mostly liquid stool; $3=$ all liquid stool), rectal bleeding $(0=$ no blood; $1=$ blood with occasional stools; 2 =blood with most stools; $3=$ blood with all stools), abdominal pain $(0=$ none; $1=$ pain that does not interfere with day to day activities; 2 =pain that interferes with day to day activities; $3=$ incapacitating pain), and urgency $(0=$ no urgency; $1=$ urgency with occasional stools; $2=$ urgency with most stools; $3=$ urgency with all stools).

Sigmoidoscopy - Sigmoidoscopic examination was performed using an Olympus CF-Type P10S flexible sigmoidoscope without sedation. Mucosal abnormality was graded on a scale of 0 to $3(0=$ normal mucosa with no friability; $1=$ erythematous granular mucosa with pinpoint friability; $2=$ coarse ulceration or pinpoint ulceration with spontaneous or induced friability; $3=$ gross ulceration with spontaneous haemorrhage). Three 'pinch' mucosal biopsy specimens were obtained from each patient at 15 to $25 \mathrm{~cm}$ from the anus. Some patients had a repeat biopsy so that more than one in vitro agent could be evaluated.

Disease actvity index was the sum of the symptom score plus the sigmoidoscopy score. The minimum possible score was 0 , the maximum, 15.

\section{CHEMILUMINESCENCE STUDIES}

Luminol enhanced chemiluminescence was used to estimate values of reactive oxygen species as previously described by us. ${ }^{1618}$ Light was detected by an EMI $9813 \mathrm{~B}$ photomultiplier in an EMI FACT $50 \mathrm{MK}$ III cooler, cooled to $-20^{\circ} \mathrm{C}$ and operated at 1375 volts. Single photon pulses were detected with an EMI APED amplifier discriminator (Thorn EMI, Ruislip, England), and these pulses were recorded with a frequency counter. The photomultiplier was operated in the single photon counting mode.

Biopsy colonic mucosal samples were suspended in $2 \mathrm{ml}$ of oxygenated Krebs-Ringer solution. The tissue suspension was mixed with the incubation mixture $(0.1 \mathrm{M} \mathrm{NaCl}, 0.05 \mathrm{M}$

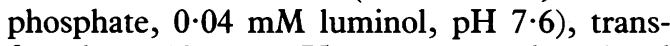
ferred to $12 \mathrm{~mm} \times 75 \mathrm{~mm}$ test tubes (total volume $=2 \mathrm{ml}$ ), and placed in the chemiluminescence spectrophotometer where light production was measured for 1 minute. At this point various drugs or vehicles were added to the incubation media. Drugs included azide (100 $\mathrm{uM}$ ), catalase ( $2 \mathrm{ug} /$ tube), MK886 $(1 \cdot 1 \mathrm{uM})$ or indomethacin (100 uM). Chemiluminescence was again measured for 1 minute. For the allopurinol studies, a $\mathrm{pH}$ value of 11 was required to ensure solubilisation of the drug. Therefore, we obtained additional mucosal biopsy specimens, divided the tissue samples in half, and placed each half in a Krebs-Ringer solution having a $\mathrm{pH} \mathrm{11,} \mathrm{one} \mathrm{with} \mathrm{and} \mathrm{one}$ without allopurinol $(100 \mathrm{uM})$. This was important as alkaline $\mathrm{pH}$ values can by themselves increase chemiluminescence. Suspensions were incubated for 5 minutes at $37^{\circ} \mathrm{C}$. Thereafter, tissues were quickly washed and placed in a mixture containing luminol $(\mathrm{pH}=7 \cdot 6)$ and photons were counted for 1 minute.

The total protein content of the tube was evaluated by the Bradford protein method. ${ }^{25}$ The chemiluminescence data are expressed as cpm per mg protein.

Luminol enhanced chemiluminescence produced by neutrophils and induced by formylmethionyl-leucyl-proline (FMLP) was also measured in a suspension of neutrophils obtained from control subjects. The effect of different concentrations of indomethacin (12.5 to $100 \mathrm{uM}$ ) on chemiluminescence was determined. Firstly, neutrophils were freshly prepared in a standard manner as previously described. ${ }^{26}$ Neutrophils $\left(1 \times 10^{6}\right.$ cells $)$ were then added to tubes containing vehicle or indomethacin ( 0 to $100 \mathrm{uM}$ ) in a total volume of $3 \mathrm{~mL}$. Formyl-methionyl-leucyl-phenylalanine (FMLP, $0.1 \mathrm{uM}$ ) or vehicle was then added. Lastly, luminol (final concentration $=100 \mathrm{uM}$ ) was added. Chemiluminescence rates were measured for 15 minutes using the same Thorn/ EMI photon counting, chemiluminescence monitor noted above.

Luminol enhanced chemiluminescence induced by the addition of hypochlorous acid to an aquaeous buffer was measured and the effect of indomethacin (100 uM) was determined. Briefly, $0.5 \mathrm{ml}$ water was placed in a series of test tubes. For control experiments, $0.1 \mathrm{ml}$ vehicle (ethanol) was then added, followed by $0.9 \mathrm{ml}$ of luminol $(4 \mathrm{uM})$. Otherwise, $0.1 \mathrm{ml}$ indomethacin dissolved in ethanol was added to a final concentration of $100 \mathrm{uM}$. Lastly, sodium hypochlorite (Aldrich) was rapidly and directly added to the measuring chamber using an auto- 
matic dispenser, diluting it to a final concentration of $7 \mathrm{uM}$. These procedures were carried out in a darkened room. Chemiluminescence was monitored for 10 seconds by the same apparatus described above.

\section{MEASUREMENT OF MYELOPEROXIDASE ACTIVITY} IN COLONIC TISSUE

Tissue samples were assessed biochemically for the neutrophil marker enzyme, myeloperoxidase, using the method of Krawisz et al.${ }^{19}$ Tissue samples were homogenised in $50 \mathrm{mM} \mathrm{KH}_{2} \mathrm{PO}_{4} /$ $\mathrm{K}_{2} \mathrm{HPO}_{4}$ buffer ( $\mathrm{pH}$ 6.0) and sonicated (30 seconds) in an ice water bath. After centrifugation $(20000 \mathrm{~g})$ for 10 minutes at $4^{\circ} \mathrm{C}$, the supernatant was discarded and $50 \mathrm{mM} \mathrm{KH}_{2} \mathrm{PO}_{4}$ $\mathrm{K}_{2} \mathrm{HPO}_{4}$ buffer containing $0.5 \%$ hexadecyltrimethylammonium bromide was added to the pellet. The mixture was homogenised for 30 seconds, diluted with $50 \mathrm{mM} \quad \mathrm{K}_{2} \mathrm{HPO}_{4} /$ hexadecyltrimethylammonium bromide $(1 \mathrm{~g} /$ $5 \mathrm{ml}$ ), and sonicated for 30 seconds in an ice bath. The enzyme activities were determined spectrophotometrically. The enzymatic reaction was started by mixing the sample $(0.1 \mathrm{ml})$ with $2.9 \mathrm{ml}$ of $50 \mathrm{mM}$ phosphate buffer ( $\mathrm{pH} 6 \cdot 0$ ) containing $0 \cdot 167 \mathrm{mg} / \mathrm{ml}$ o-dianisidine dihydrochloride and $0.0005 \%$ hydrogen peroxide. The change in absorbance at $460 \mathrm{~nm}$ was measured with a Beckman DU 7 spectrophotometer (Beckman Instruments, Fullerton, CA). Data are expressed as the change in absorbance $(460 \mathrm{~nm})$ between 0 and 2 minutes. Myeloperoxidase activity data are presented as units per mg protein.

\section{STATISTICAL ANALYSES}

For chemiluminescence, the Student's $t$ test was used to detect statistically significant differences between groups. When the effects of drugs were assessed, a Student paired $t$ test was used. Correlation data were analysed using the Spearman rank correlation method. Data are presented as mean (SEM). A result was considered statistically significant if $\mathrm{p} \leq \mathbf{0} \cdot 05$.

\section{Results}

Luminol enhanced chemiluminescence was significantly raised in inflamed mucosa from patients with ulcerative colitis (Fig 1). The chemiluminescence signal significantly correlated with the severity of colitis as assessed by sigmoidoscopic score (Fig 2A), symptom score (Fig 2B) or disease activity index (Fig 2C). Sigmoidoscopic and symptom scores also

Figure 1: Comparison of colonic mucosal chemiluminescence in subjects with or without ulcerative colitis. Chemiluminescence from inflamed colonic mucosa of patients with ulcerative patients with ulcerative
colitis was significantly $\left.{ }^{\star}\right)$ raised compared with colonic mucosa from control subjects.
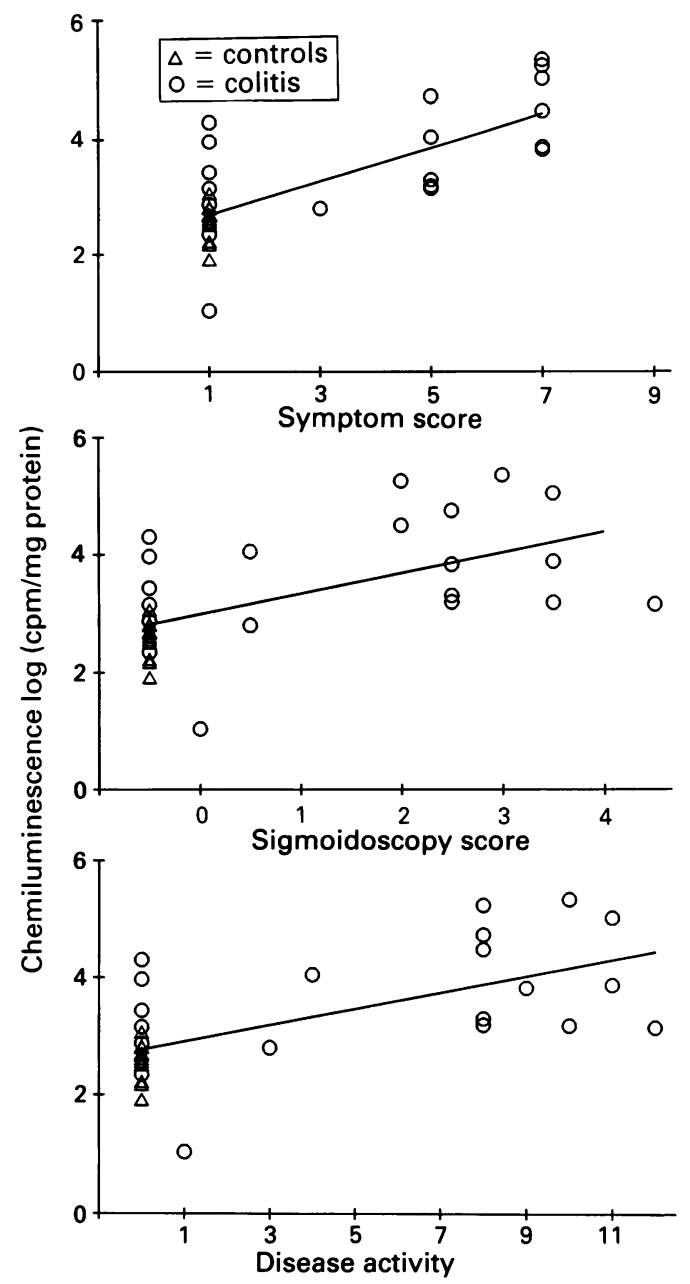

Figure 2: Correlation between chemiluminescence from colonic mucosa and symptom score $(A)$, sigmoidoscopy score $(B)$, and disease activity $(C)$. Chemiluminescence from colonic mucosa correlated positively and significantly (Spearman rank correlation) with symptom score, sigmoidoscopy score, and disease activity. Disease activity = symptom score plus sigmoidoscopy score. Note that the chemiluminescence score in (A) for subjects with sigmoidoscopy scores of zero includes both subjects with colitis $(n=10)$ but with inactive disease as well as controls $(n=14)$.

showed a significant correlation with each other $(\mathrm{r}=0.91)$.

As expected, mucosal myeloperoxidase activity, an index of mucosal neutrophil infiltration, was also raised in patients with colitis (Fig 3). Myeloperoxidase activity correlated significantly with disease activity (Fig 4) and with chemiluminescence (Fig 5).

To determine the contribution of myeloper-

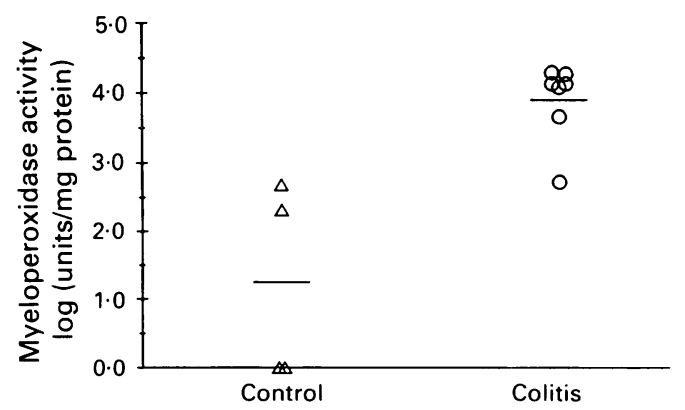

Figure 3: Comparison of myeloperoxidase activity in colonic mucosa from subjects with and without ulcerative colitis. Myeloperoxidase in inflamed colonic mucosa of patients with ulcerative colitis was significantly $\left(^{\star}\right)$ raised compared with subjects with normal colonic mucosa. 
Figure 4: Correlation between myeloperoxidase activity of colonic mucosa and disease activity. There was a significant and positive correlation between myeloperoxidase activity and disease activity.
Figure 5: There was a significant and positive correlation between chemiluminescence and the myeloperoxidase activity of colonic mucosa. oxidase in mucosal chemiluminescence, an inhibitor of myeloperoxidase activity, azide, was added to the colonic mucosal preparation in vitro. Azide significantly inhibited $(-76 \%)$ the chemiluminescence signal (Fig 6). To determine the contribution of $\mathrm{H}_{2} \mathrm{O}_{2}$, catalase, a scavenger of $\mathrm{H}_{2} \mathrm{O}_{2}$, which is the substrate for the myeloperoxidase reaction, was added. Catalase also inhibited (-78\%) chemiluminescence (Fig 6).

By contrast, the lipoxygenase inhibitor, MK886, did not significantly affect chemiluminescence (Fig 6). Also, allopurinol, an inhibitor of xanthine oxidase did not significantly affect chemiluminescence (Fig 6).

A cyclo-oxygenase inhibitor, indomethacin, also significantly decreased the chemiluminescence signal but the magnitude $(-34 \%)$ was substantially less than the effects of either azide or catalase (Fig 6).
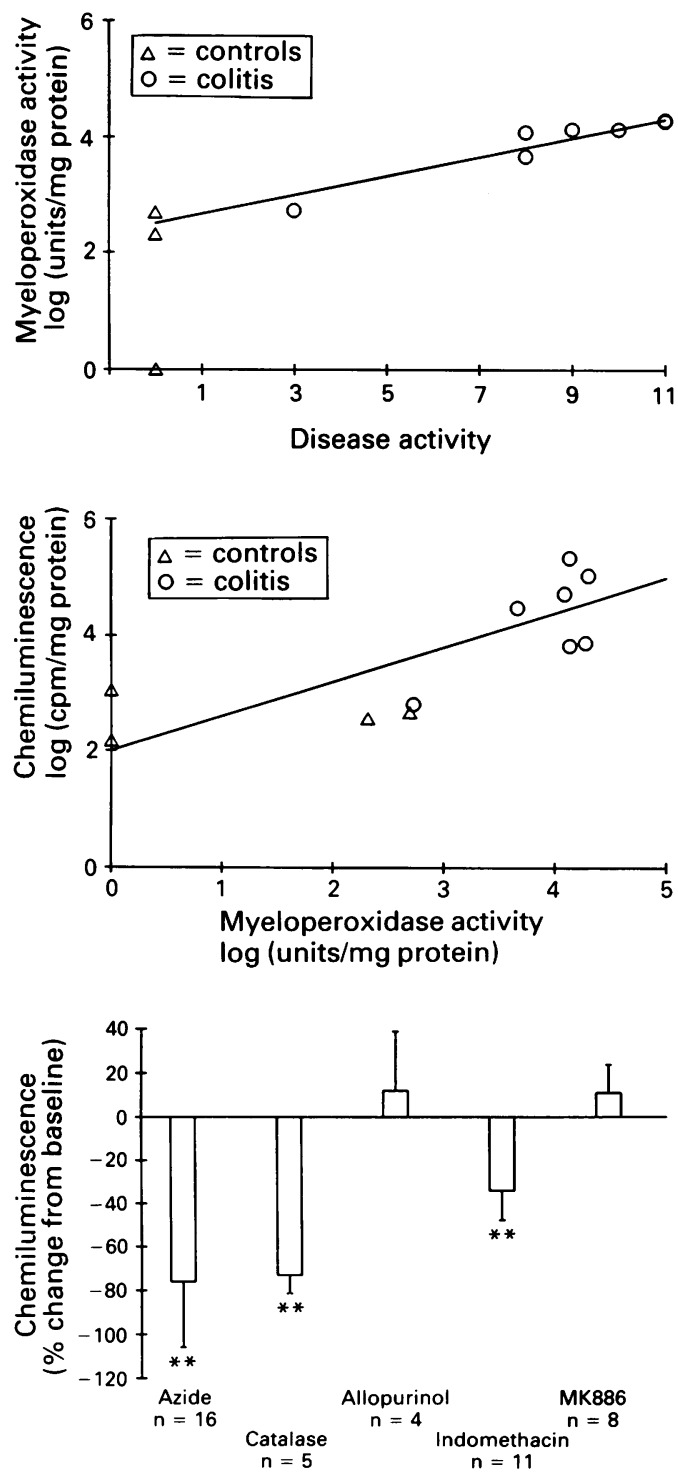

Figure 6: The effect of in vitro addition of pharmacological agents on chemiluminescence from colonic mucosa. Final drug concentrations were: for the myeloperoxidase inhibitor azide $(100 \mathrm{uM})$, the $\mathrm{H}_{2} \mathrm{O}_{2}$ scavenger catalase (2 ug), lipoxygenase $(100 \mathrm{uM})$, the $\mathrm{H}_{2} \mathrm{O}_{2}$ scavenger catalase (2 ug), lipoxyg
inhibitor MK886 $(1 \cdot 1 \mathrm{uM})$, cyclo-oxygenase inhibitor indomethacin $(100 \mathrm{uM})$, and the xanthine oxidase inhibitor allopurinol $(100 \mathrm{uM})$. The data are presented as percentage change from chemiluminescence values from inflamed colonic mucosa before agents were added. ${ }^{\star} \star$ Represents a mean value whose difference from the control mean was statistically significant at the $p<0.01$ value.

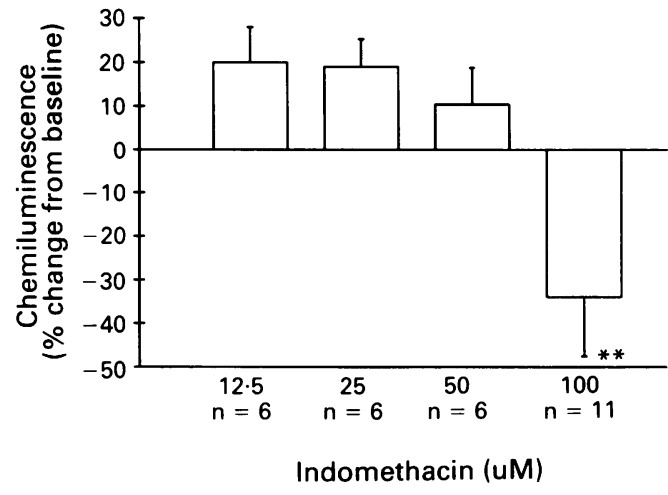

Figure 7: Effect of indomethacin on chemiluminescence in colonic biopsies. Final indomethacin concentrations are shown in the figure. The data are presented as percentage change from chemiluminescence values from inflamed colonic mucosa before indomethacin was added. ${ }^{\star}{ }^{2}$ Represents a mean value whose difference from the control mean was statistically significant at the $p<0.01$ value.

To find out if indomethacin inhibited chemiluminescence through its ability to inhibit the cyclo-oxygenase enzyme or its ability to scavenge oxygen free radicals, a dose response experiment was done. Indomethacin, at doses that are known to inhibit cyclo-oxygenase, ${ }^{27}$ failed to inhibit chemiluminescence production by the inflamed colonic mucosa (Fig 7). Furthermore, we evaluated the effect of indomethacin on chemiluminescence production by neutrophil after stimulation by FMLP, a system that is not dependent on cyclo-oxygenase. ${ }^{28}$

We showed that indomethacin, dose dependently, inhibited chemiluminescence elicited by stimulation of a suspension of neutrophils by FMLP (Fig 8). To discover if the inhibitory effect of indomethacin is because of its ability to scavenge $\mathrm{HOCl}$, we evaluated the effect of indomethacin on $\mathrm{HOCl}$ production. Indomethacin, at $100 \mathrm{uM}$, significantly inhibited chemiluminescence produced by addition of $\mathrm{HOCl}$ in the presence of luminol. The mean cpm in control tubes was $96588(n=4, S E M=$ $14117)$. The mean counts when indomethacin was present was $39417(n=4, S E M=13255)$. Indomethacin thus elicited a $61 \%$ decrease in chemiluminescence $(p=0 \cdot 011)$.

\section{Discussion}

The most disabling feature of ulcerative colitis, a chronic relapsing disease, is episodic acute exacerbation. During these periods, there is an association between acute inflammation (presence of neutrophils), tissue injury, and signs and symptoms of active disease. During intervals of remission, subjects are asymptomatic despite the fact that the mucosa is not entirely normal and the putative causal factor(s) are still present. Hence, identification and characterisation of the mediators that lead to the acute inflammation and tissue injury could have a significant impact on clinical management of patients with colitis.

Among the putative mediators are reactive oxygen species. Reactive oxygen species seem to play a part in inflammation and tissue injury in a number of diseases ${ }^{3-5}$ and have recently been implicated in the inflammation and tissue injury 


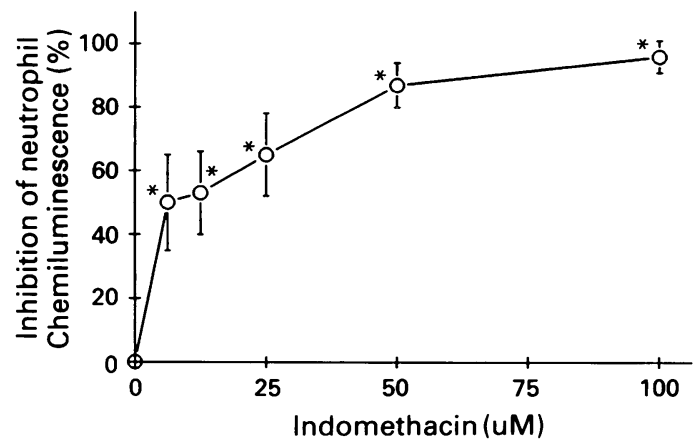

Figure 8: Effect of indomethacin on chemiluminescence elicited by the stimulation of neutrophils by formyl-methionylleucyl-phenylanine (FMLP). FMLP was added to a suspension of neutrophils to elicit increases in chemiluminescence. Error bars are mean $(S E M) .{ }^{\star}$ Represents a mean value whose difference from the control $(0 \mathrm{uM})$ indomethacin mean was statistically significant at the $p<0.05$ value.

in the gastrointestinal tract. ${ }^{56812}$ Additionally, drugs widely used for the treatment of ulcerative colitis such as sulfasalazine and 5-ASA are known to be potent anti-reactive oxygen species agents. $^{729}$

This study presents evidence, based on chemiluminescence data, that further suggests that reactive oxygen species play a part in ulcerative colitis. We showed that luminol enhanced chemiluminescence from colonic mucosal biopsy specimens is increased in patients with ulcerative colitis. Our finding is similar to that of Simmonds et $a l^{17}$ who also showed an increase in chemiluminescence in ulcerative colitis. This increase in chemiluminescence is not specific to ulcerative colitis in itself as we have also shown that inflammation of the colon from other causes will also increase chemiluminescence. For example, we showed that colonic inflammation induced in rats by acetic acid $^{16}$ or by mitomycin-c ${ }^{16} 18$ is also associated with an increase in chemiluminescence. Additionally, Simmonds $e a{ }^{17}$ showed increased chemiluminescence in the inflamed colonic mucosa of patients with Crohn's disease.

This study, which shows a positive correlation between chemiluminescence and disease activity (sigmoidoscopy, symptoms) suggests that reactive oxygen species play a part in the severity of the inflammation and tissue injury in ulcerative colitis. Our data suggest, then, that chemiluminescence may be useful as a new marker when determination of the disease activity of ulcerative colitis is desired. This study confirms our initial preliminary report on increased chemiluminescence in ulcerative colitis in humans ${ }^{16}$ and the work of Simmonds et al, ${ }^{17}$ as they too showed a positive correlation between chemiluminescence and disease activity even though they based disease activity solely on sigmoidoscopy score.

Information on the cellular and biochemical sources of reactive oxygen species would be useful as this could lead to a specific target or targets for development of new treatment methods. Although the source of the light production giving rise to the chemiluminescence signal cannot be determined definitively, ${ }^{1030-33}$ our present data and data from Simmonds $e t a l^{17}$ suggest that increases in reactive oxygen species generated by neutrophils are in fact an important source. Our findings that (1) myeloperoxidase activity correlates with disease activity and (2) azide - at a concentration that selectively inhibits myeloperoxidase - inhibits chemiluminescence, suggest that the neutrophils are an important cellular source of the chemiluminescence. These findings are consistent with the data of Simmonds $e t a l^{17}$ although they used myeloperoxidase staining rather than measurement of myeloperoxidase activity, and the concentration of azide they used was 10-fold higher than concentrations that are believed to be specific for myeloperoxidase inhibition and thus may have been somewhat non-selective.

Whatever the cellular origin of the chemiluminescence, it is also important to identify the biochemical reaction(s) that produce the precursors for the myeloperoxidase reaction that produces hypochlorous acid. It is generally accepted that superoxide anion is most frequently the initial reactive oxygen species that results in production of a cascade of other reactive oxygen species such as hydroxyl radical, $\mathrm{H}_{2} \mathrm{O}_{2}$, and hypochlorous acid. ${ }^{3}$ These three reactive oxygen species are believed to be responsible for tissue injury. ${ }^{3}$

One possible source for superoxide is the xanthine/xanthine oxidase reaction. This reaction has been implicated in the generation of reactive oxygen species in ischemic reperfusion injury, ${ }^{2021}$ a mechanism which has been recently postulated for inflammatory bowel disease. Our data do not support the idea that the xanthine/ xanthine oxidase pathway plays a part in ulcerative colitis as allopurinol, in concentrations known to inhibit xanthine oxidase activity, ${ }^{84}$ did not affect chemiluminescence.

Another possible source of superoxide anion is the oxidation of arachadonic acid by the lipoxygenase or cyclo-oxygenase pathways. It is well known that products of the cyclo-oxygenase reaction, prostaglandins, and of the lipoxygenase reaction, leukotrienes, are increased in inflammatory bowel disease. ${ }^{35}$ It is postulated that the deleterious effect of these reactions and their products is, at least in part, through their ability to generate reactive oxygen species. ${ }^{15} \mathrm{We}$ now show that indomethacin, a cyclo-oxygenase inhibitor, at $100 \mathrm{uM}$ decreases chemiluminescence. This finding suggests that the cyclooxygenase pathway could be an important contributor to the production of reactive oxygen species in the inflamed mucosa. As indomethacin is also known to be an reactive oxygen species scavenger, ${ }^{22}$ however, its effect may be independent of its inhibition of cyclo-oxygenase. Indeed, indomethacin failed to inhibit chemiluminescence when added in vitro to colonic biopsy specimens at concentrations below $100 \mathrm{uM}$ despite a maximum inhibition at $0.08 \mathrm{uM}$ of cyclo-oxygenase in cell free systems. ${ }^{27}$ Moreover, we showed that indomethacin inhibited chemiluminescence elicited by stimulation of a suspension of neutrophils by FMLP, a system in which oxygen free radicals are not thought to derive from the cyclo-oxygenase reaction. ${ }^{28}$ Furthermore, PGE type compounds have been shown to 
be effective inhibitors of FMLP activated human neutrophils, ${ }^{29}$ making it even more unlikely that indomethacin induced inhibition of cyclooxygenase underlies the ability of indomethacin to attenuate FMLP induced chemiluminescence from neutrophils.

Hence, our data strongly suggest that the inhibiting effects of indomethacin is not through cyclo-oxygenase inhibition. Cyclo-oxygenase pathway does not seem to significantly contribute to chemiluminescence production by the inflamed mucosa.

Finally, we showed that indomethacin at a concentration of $100 \mathrm{uM}$ significantly inhibited the chemiluminescence produced chemically by the interaction of hypochlorous acid with luminol. These data together show that indomethacin scavenges oxygen free radicals.

Although it was initially believed that lowering mucosal prostaglandin values in ulcerative colitis would be therapeutic, and that the beneficial effect of therapeutic agents sulfasalazine and 5-ASA analogues is through their ability to inhibit cyclo-oxygenase, it is now believed that a simple decrease in prostaglandins does not elicit a beneficial effect in ulcerative colitis and may even be harmful. ${ }^{36-39}$ This conclusion is based on the fact that potent prostaglandin synthesis inhibitors such as indomethacin not only do not improve ulcerative colitis, but also can worsen the disease activity, ${ }^{36-39}$ implying that prostaglandins have some protective or repair ability, or both.

These data suggest that, although high values of reactive oxygen species may be harmful or injurious, compounds such as indomethacin, which effectively decrease both reactive oxygen species and prostaglandins, may not be beneficial in the treatment of ulcerative colitis.

Another possible source of superoxide anion is oxidation of arachadonic acid by lipoxygenase. Our data, which show that leukotriene inhibitors do not decrease chemiluminescence, suggest that the lipoxygenase pathway does not significantly contribute to the production of reactive oxygen species by the inflamed colonic mucosa. The known clinical benefit of leukotriene inhibitors in ulcerative colitis,${ }^{+0}$ however, could still be due to their ability to indirectly decrease tissue reactive oxygen species. For example, it is known that leukotrienes are potent chemotactic compounds. ${ }^{41+2}$ If, as we believe, the main source of mucosal reactive oxygen species in inflammation is the neutrophil, then leukotriene synthesis inhibitors could prevent aggregation of neutrophils in the mucosa, thus decreasing reactive oxygen species and tissue injury.

We conclude that (1) reactive oxygen species are generated in increased amounts in inflamed mucosa in patients with ulcerative colitis; (2) these increased values of reactive oxygen species may be an important contributor to the tissue injury in ulcerative colitis; (3) the most likely cellular source of reactive oxygen species in ulcerative colitis is the neutrophil; (4) a likely culprit in the tissue injury is a product of the myeloperoxidase reaction, namely hypochlorous acid; (5) arachadonic acid pathways, including cyclo-oxygenase and lipoxygenase, probably do not significantly contribute to production of reactive oxygen species by the inflamed mucosa.

This study was supported in part by a BRSG grant from Loyola University Medical School. MK886 was the generous gift from the Merck Frosst Center for Therapeutic Research (Canada). We wish to thank Ms Mary McLennon for assistance with preparation of the manuscript.

Some of the data contained in this manuscript have been published in abstract form in Gastroenterology 1992; 103: A693, 1993; 104: A778 and in Clin Res 1992; 40: 419A.

1 Kirsner JB, Shorter RG. Recent developments in 'non-specific inflammatory bowel disease. $N$ Engl $\mathcal{F}$ Med 1982; 306: 775 84

2 McDonaldson RM. In: Sleisenger M, ed. Gastrointestinal disease. Philadelphia: WB Saunders, 1989: 1327-58.

3 Weiss SJ. Tissue destruction by neutrophils. $N$ Engl $\mathcal{F}$ Med 1989; 320: 365-76.

4 Cross CE, Halliwell B, Borish ET, Pryor WA, Ames BN, Sau $\mathrm{KL}$, et al. Oxygen radicals and human disease. Ann Intern Med 1987; 107:526-45.

5 Parks D, Buckley G, Granger N. Role of oxygen free radicals in digestive tract disease. Surgery 1983; 94: 415-22.

6 Harris ML, Schiller HJ, Reilly PM, Donowitz M, Grisham $M B$, Buikley GB. Free radicals and other reactive oxygen metabolites in inflammatory bowel disease: cause, consequence or epiphenomenon? Pharmacol Ther 1992; 53. 375-408.

7 Williams JG, Hallett MB. Effect of sulfasalazine and its active metabolite, 5-aminosalicylic acid, on toxic oxygen metabolite production by neutrophils. Gut 1989; 30: 1581-7.

8 Keshavarzian A, Morgan G, Sedghi S, Gordon JH, Doria M Role of reactive oxygen metabolites in experimental colitis. Gut 1990; 31: 786-90.

9 Boveris A, Cadenas E, Reiter R, Filipowski M, Nakase Y, Chance B. Organ chemiluminescence: Non-invasive assay Chance B. Organ chemiluminescence: Non-invasive assay 1980; 77: 347-51.

10 Stevens P. Lucigenin and luminol-dependent chemiluminescence to selectively differentiate the oxidative pathways of phagocytic cells. In: Van Dyke K, Castranova V, eds Cellular chemiluminescence. Boca Raton, Florida, CRC Press, 1987: 105-12.

11 Rao PS, Luber JM, Milinowicz J, Lalezari P, Mueller HS Specificity of oxygen radical scavengers and assessment of free radical scavenger efficiency using luminol-enhanced chemiluminescence. Biochem Biophys Res Commun 1988; 150: $39-44$.

12 Suematsu M, Suzuki M, Kitahora T, Miura S, Susuki K, Mibi $\mathrm{T}$, et al. Increased respiratory burst of leukocytes in inflammatory bowel disease. The analysis of free radicals inflammatory bowel disease. The analysis of free radicals generation by using a chem

13 Boveris A, Cadenas E, Chance B. Ultraweak chemiluminescence: A sensitive assay for oxidative radical reactions. $\mathrm{Fed}$ Proc 1980; 40: 195-8.

14 Roldan EJA, Pinus CR, Turrens JF, Boveris A. Chemiluminescence of ischemic and reperfused intestine in vivo. Gu 1989; 30: 184-7.

15 Craven PA, Pfanstiel J, DeRubertis FR. Role of reactive oxygen in bile salt stimulation of colonic epithelial proliferation. 7 Clin Invest 1986; 77: 850-9.

16 Keshavarzian A, Sedghi A, Kanofsky J, List T, Robinson C, Ibrahim $\mathrm{C}$, et al. Excessive production of reactive oxygen metabolites by inflamed colon: Analysis by chemiluminemetabolites by inflamed colon: Analysis by chemil

17 Simmonds NJ, Allen RE, Stevens TRJ, Van Someren RNM Blake DR, Rampton DS. Chemiluminescence assay of Blake DR, Rampton DS. Chemiluminescence assay of mucosal reactive oxygen metabolites in inflam
disease. Gastroenterology 1992; 103: 186-96.

18 Keshavarzian A, Doria MI, Sedghi S, Kanofsky JR, Hecht D, Holmes EW, et al. Mitomycin-induced colitis in rats. A new animal model of acute colonic inflammation implicatin reactive oxygen species. $\mathcal{F}$ Lab Clin Med 1992; 120: 778-91.

19 Krawisz JE, Sharon P, Stenson WF. Quantitative assay fo acute intestinal inflammation based on myeloperoxidase activity. Gastroenterology 1984; 87: 1344-50.

20 Granger N, McCord J, Parks D, Hollwarth M. Xanthine oxidase inhibitors attenuate ischemia-induced vascular permeability changes in the cat intestine. Gastroenterolog 1986; 90: $80-4$.

21 Parks D, Granger N. Ischemic-induced vascular changes: Role of xanthine oxidase and hydroxyl radicals. Am $\mathcal{F}$ Physiol 1983; 245: G285-9.

22 Pekoe G, Van Dyke K, Mengoli H, Peden D, English D. Comparison of the effects of antioxidant non-steroidal antiinflammatory drugs against myeloperoxidase and hypochlorous acid luminol-enhanced chemiluminescence. Agents Actions 1982; 12: 1-2.

23 Karayalcin SS, Sturbaum W, Wachsman JT, Cha JH, Powell DW. Hydrogen peroxide stimulates rat colonic prostaglandin production and alters electrolyte transport. $\mathcal{F}$ Clin Invest 1990; 86: 60-8.

24 Sedor JR. Free radicals and prostanoid synthesis. $\mathcal{F}$ Lab Clin Med 1986; 108: 521 .

25 Bradford MM. A rapid and sensitive method for the quantitation of microgram quantities of protein utilizing the principle of protein-dye binding. Anal Biochem 1976; 72: 248-54.

26 Boyum A. Isolation of mononuclear cells and granulocytes from human blood. Isolation of mononuclear cells by one centrifugation and of granulocytes by combining centrifuga- 
tion and sedimentation at $1 \times \mathrm{g}$. Scand $\mathcal{F}$ Clin Lab Invest Suppl 1968; 97: 77-89.

27 Kulmacz RJ. Topography of prostaglandin $\mathrm{H}$ synthase. Antiinflammatory agents and the protease-sensitive arginine 253 region. $\mathcal{F}$ Biol Chem $1989 ; 264: 14136-44$.

28 Dahlgren $C$. Effect of different inhibitors on the intracellularly and extracellularly generated chemiluminescence induced by formylmethionyl-leucyl-phenylalanine in polymorphoby formylmethionyl-leucyl-phenylalanine in polymorpho-
nuclear leukocytes. Cellular response in the presence of nuclear leukocytes. Cellular response in the presence of
mannitol, benzoate, taurine, indomethacin and NDGA mannitol, benzoate, taurine, indome

29 Hecker G, Ney P, Schror K. Cytotoxic enzyme release and oxygen centred radical formation in human neutrophils are selectively inhibited by E-type prostaglandins but not by PGI-2. Naunyn Schmeidebergs Arch Pharmacol 1990; 341 : 308-15.

30 Aruoma OL, Wasil M, Halliwell B, Hoey BM, Butler J. The scavenging of oxidants by sulfasalazine and its metabolites: A possible contribution to their anti-inflammatory effects. Biochem Pharmacol 1987; 36: 3739-42.

31 Dahlgren C, Stendahl O. Role of myeloperoxidase in luminoldependent chemiluminescence of polymorphonuclear leukocytes. Infect Immun 1983; 39: 736-41.

Dechatelet LR, Long GD, Shirley PS, Bass DA, Thomas MJ Henderson FW, et al. Mechanism of the luminol-dependen chemiluminescence of human neutrophils. F Immunol 1982; 129: 1589-93

33 Merenyi G, Lind J, Eriksen TE. The reactivity of superoxide and its ability to induce chemiluminescence with luminol. Photochem Photobiol 1985; 41: 203-8.
34 Elion GB. Enzymatic and metabolic studies with allopurinol. Ann Rheum Dis 1966; 25: 608-14.

35 Schumbert R, Towner J, Zipser RD Role of eicosandoids in human and experimental colitis. Dig Dis Sci 1988; 33: 58$64 S$.

36 Rampton DS, Sladen GE. Prostaglandin synthesis inhibitors in ulcerative colitis: Flurbiprofen compared with convenin ulcerative colitis: Flurbiprofen compared with

37 Rampton DS, Sladen GE. Relapse of ulcerative proctocolitis during treatment with nonsteroidal anti-inflammatory drugs.

38 Berry H, Swinson D, Jones H, Hamilton EBD. Indomethacin and naproxen suppositories in the treatment of rheumatoid arthritis. Ann Rheum Dis 1978; 37: 370-3.

39 Wright V, Hopkins R. A note on indomethacin suppositorie in the treatment of rheumatic conditions. Rheumatol Rehabi $1979 ; 18: 186-7$.

40 Stenson WF, Lauritsen K, Laursen LS, Rask-Madsen J, Jacobsen O, Naesdal D, et al. A clinical trial of zileuton, a specific inhibitor of 5-lipoxygenase in ulcerative colitis specific inhibitor of 5-lipoxygen
Gastroenterology 1991; 100: A253.

41 Nielsen $\mathrm{OH}$, Ahnfelt-Ronne I, Elmgreen J. Abnormal metabolism of arachidonic acid in chronic inflammatory bowe disease: enhanced release of leukotriene $\mathrm{B}_{4}$ from activated neutrophils. Gut 1987 ; 28

42 Lehr HA, Hubner C, Finckh B, Angermuller S, Nolte D Beisiegel $U$, et al. Role of leukotrienes in leukocyte adhesion following systemic administration of oxidatively modified human low density lipoprotein in hamsters. $\mathcal{F}$ Clin Inves 1991; 88: 9-14. 